\title{
Ein Spektralabbildungssatz
}

\author{
Von
}

CH. SCHMOEGER

1. Einleitung. In dieser Arbeit bezeichne $X$ einen komplexen Banachraum, $L(X)$ die Banachalgebra der stetigen Endomorphismen auf $X$ und $I$ die identische Abbildung auf $X$. Mit $N(T), T(X), \sigma(T)$ und $\varrho(T)$ bezeichnen wir beziehentlich Nullraum, Bildraum, Spektrum und Resolventenmenge des Operators $T \neq 0$ in $L(X)$.

T. Kato zeigt in [3] (Theorem 3, S. 297), daß die Menge

$$
\begin{array}{r}
\varrho_{K}(T):=\{\lambda \in \mathbb{C}:(\lambda I-T)(X) \text { ist abgeschlossen und } \\
\left.N(\lambda I-T) \subseteq \bigcap_{n=1}^{\infty}(\lambda I-T)^{n}(X)\right\}
\end{array}
$$

eine offene Teilmenge der komplexen Ebene ist. Man beachte hierbei, daß die Katosche Bedingung $v(\lambda I-T: I)=\infty$ gleichbedeutend mit $N(\lambda I-T) \subseteq \bigcap_{n=1}^{\infty}(\lambda I-T)^{n}(X)$ ist
(s. [3], S. 290).

Da $\varrho_{K}(T)$ die Resolventenmenge $\varrho(T)$ umfaßt, ist das Komplement

$$
\sigma_{K}(T):=\mathbb{C} \backslash \varrho_{K}(T)
$$

eine kompakte Teilmenge des Spektrums $\sigma(T)$. In Satz 2 der vorliegenden Arbeit werden wir sehen, daß $\sigma_{K}(T)$ stets nicht leer ist.

Ziel dieser Arbeit ist es, einen Spektralabbildungssatz für die Menge $\sigma_{K}(T)$ zu beweisen.

Welche Rolle die Menge $\varrho_{K}(T)$ in der Operatorentheorie spielt, soll nun geschildert werden.

K. H. Förster beweist in [1] (Theorem 3) den folgenden

Satz 1. In jeder Zusammenhangskomponente von $\varrho_{K}(T)$ sind die Räume

$$
\overline{\bigcup_{n=1}^{\infty} N\left((\lambda I-T)^{n}\right)} \text { und } \bigcap_{n=1}^{\infty}(\lambda I-T)^{n}(X)
$$

unabhängig von $\lambda$. 
Bekanntlich nennt man $\lambda_{0} \in \mathbb{C}$ einen Semifredholmpunkt von $T$, wenn $\beta\left(\lambda_{0} I-T\right):=\operatorname{codim}\left(\lambda_{0} I-T\right)(X)<\infty$ oder $\alpha\left(\lambda_{0} I-T\right):=\operatorname{dim} N\left(\lambda_{0} I-T\right)<\infty$ und $\left(\lambda_{0} I-T\right)(X)$ abgeschlossen ist. Nach Theorem 6 in [3] (S. 316) gilt nun: Die Menge der Semifredholmpunkte von $T$ ist offen, und in jeder Zusammenhangskomponente $\Gamma$ dieser Menge sind die Defekte $\alpha(\lambda I-T)$ und $\beta(\lambda I-T)$ konstant, mit Ausnahme einer höchstens abzählbaren Menge, die in $\Gamma$ keine Häufungspunkte besitzt. Außerdem ist der Index von $\lambda I-T$, also die Größe $\alpha(\lambda I-T)-\beta(\lambda I-T)$, konstant. Daher ist für einen Semifredholmpunkt $\lambda_{0}$ die folgende Definition sinnvoll (vgl. [4]):

$$
j\left(\lambda_{0}\right):= \begin{cases}\alpha\left(\lambda_{0} I-T\right)-\alpha(\lambda I-T), & \text { falls } \alpha\left(\lambda_{0} I-T\right)<\infty \\ \beta\left(\lambda_{0} I-T\right)-\beta(\lambda I-T), & \text { falls } \beta\left(\lambda_{0} I-T\right)<\infty,\end{cases}
$$

wobei $\lambda \neq \lambda_{0}$ in einer hinreichend kleinen Umgebung von $\lambda_{0}$ liegen soll.

Aus Theorem 3 und Theorem 5 in [3] (S. 298 bzw. 315) folgt, daß für einen Semifredholmpunkt $\lambda_{0}$ gilt:

$$
j\left(\lambda_{0}\right)=0 \Leftrightarrow \lambda_{0} \in \varrho_{K}(T) .
$$

Die geschilderten Eigenschaften der Menge $\varrho_{K}(T)$ zeigen, daß man ihre Punkte in einem gewissen Sinne als „gutartig“ betrachten kann.

2. Hilfsmittel. Einen Beweis des ersten Hilfssatzes findet man in [2] (Satz 80.1 c)).

Hilfssatz 1. Sind $\lambda_{1}$ und $\lambda_{2}$ zwei verschiedene komplexe Zahlen, so gilt:

$$
\bigcup_{n=1}^{\infty} N\left(\left(\lambda_{1} I-T\right)^{n}\right) \subseteq \bigcap_{n=1}^{\infty}\left(\lambda_{2} I-T\right)^{n}(X)
$$

Hilfssatz 2. Sind $\lambda_{1}, \ldots, \lambda_{k}$ paarweise verschiedene komplexe Zahlen und $n_{1}, \ldots, n_{k} \in \mathbb{N}$, dann gilt für das Polynom $p(\lambda):=\prod_{i=1}^{k}\left(\lambda-\lambda_{i}\right)^{n_{i}}$ :

$$
N(p(T))=N\left(\left(T-\lambda_{1} I\right)^{n_{1}}\right) \oplus \cdots \oplus N\left(\left(T-\lambda_{k} I\right)^{n_{k}}\right)
$$

und

$$
p(T)(X)=\bigcap_{i=1}^{k}\left(T-\lambda_{i} I\right)^{n_{i}}(X)
$$

B e w e i s. Die erste Aussage ergibt sich aus Satz 80.1 a) in [2], während man die zweite mit Aufgabe 80.5 in [2] einsehen kann.

Den folgenden Hilfssatz haben wir [3] entnommen (Lemma 511 (S. 289) und die daran anschließenden Bemerkungen). 
Hilfssatz 3. Für $\lambda \in \mathbb{C}$ sind die folgenden Aussagen äquivalent:

(a) $N(\lambda I-T) \subseteq \bigcap_{n=1}^{\infty}(\lambda I-T)^{n}(X)$.

(b) $\bigcup_{n=1}^{\infty} N\left((\lambda I-T)^{n}\right) \subseteq(\lambda I-T)(X)$.

(c) $\bigcup_{n=1}^{\infty} N\left((\lambda I-T)^{n}\right) \subseteq \bigcap_{n=1}^{\infty}(\lambda I-T)^{n}(X)$.

3. Eigenschaften von $\sigma_{K}(T)$. Für eine Menge $M \subseteq \mathbb{C}$ sei $\partial M$ die Menge ihrer Randpunkte.

\section{Satz 2.}

(a) Ist $C$ eine Zusammenhangskomponente des Spektrums $\sigma(T)$, so gilt $\partial C \leqq \sigma_{K}(T)$.

(b) $\partial \sigma(T) \subseteq \sigma_{K}(T)$.

Insbesondere ist also $\sigma_{K}(T) \neq \emptyset$ und jede Zusammenhangskomponente von $\sigma(T)$ trifft $\sigma_{K}(T)$.

B e w e is. (a) Wir nehmen an, für ein $\lambda_{0} \in \partial C$ gelte $\lambda_{0} \in \underline{Q}_{K}(T)$. Es bezeichne $K$ diejenige Komponente von $\varrho_{K}(T)$, für die $\lambda_{0} \in K$ gilt. Wegen $\lambda_{0} \in \partial \sigma(T)$ und der Offenheit von $K$ bekommen wir $K \cap \varrho(T) \neq \emptyset$. Hieraus erhält man mit Satz 1

$$
N(\lambda I-T)=\{0\} \quad \text { und }(\lambda I-T)(X)=X \quad \text { für jedes } \lambda \in K,
$$

woraus sich wegen $\lambda_{0} \in K$ der Widerspruch $\lambda_{0} \in \varrho(T)$ ergibt.

(b) ergibt sich aus (a) und der Beziehung $\partial C=C \cap \partial \sigma(T)$ für jede Zusammenhangskomponente $C$ von $\sigma(T)$.

Ist $T \in L(X)$, so sei $H(T)$ die Menge aller komplexwertigen Funktionen $f$, die auf einer offenen Umgebung $\Delta(f)$ des Spektrums holomorph sind. Der Operator $f(T)$ für $f \in H(T)$ ist dann durch den bekannten Riesz-Dunfordschen Funktionalkalkül erklärt (s. [2], §99).

Der nächste Satz wird uns beim Beweis des Spektralabbildungssatzes von Nutzen sein.

Satz 3. Ist $g \in H(T)$ und verschwindet $g$ in keinem Punkt von $\sigma_{K}(T)$, so besitzt $g$ in $\sigma(T)$ höchstens endlich viele Nullstellen.

B e w e is. Wir nehmen an, die Behauptung sei falsch. Dann besitzt die Nullstellenmenge von $g$ einen Häufungspunkt $\lambda_{0}$ in $\sigma(T)$. Wir bezeichnen mit $C$ die Zusammenhangskomponente von $\sigma(T)$, die den Punkt $\lambda_{0}$ enthält und mit $K$ diejenige Komponente des Definitionsbereiches von $g$, für die $\lambda_{0} \in K$ gilt. Dann ist $C \subseteq K$ und $g \equiv 0$ auf $K$. Aus Satz 2 erhalten wir $C \cap \sigma_{K}(T) \neq \emptyset$, also auch $K \cap \sigma_{K}(T) \neq \emptyset$. Somit besitzt $g$ doch Nullstellen in $\sigma_{K}(T)$, im Widerspruch zur Voraussetzung.

\section{Eigenschaften der Menge $\varrho_{K}(T)$.}

Satz 4. Für $\lambda \in \varrho_{K}(T)$ und $n \in \mathbb{N}$ ist der Bildraum von $(\lambda I-T)^{n}$ abgeschlossen. 
B e we is. Ohne Beschränkung der Allgemeinheit sei $\lambda=0$. Wir zeigen die Abgeschlossenheit von $T^{n}(X)$ induktiv. $T(X)$ ist nach Voraussetzung abgeschlossen. Für ein $n \in \mathbb{N}$ sei die Abgeschlossenheit von $T^{n}(X)$ schon gezeigt. Setzt man $M:=T(X)$, so folgt aus $N(T) \subseteq \bigcap_{k=1}^{\infty} T^{k}(X)$ und Hilfssatz 3 die Inklusion $N\left(T^{n}\right) \subseteq M$, woraus sich $M+N\left(T^{n}\right)=M$ ergibt. Damit ist die Summe $M+N\left(T^{n}\right)$ abgeschlossen. Wendet man Lemma 311 aus [3] (S. 274) auf den Operator $T^{n}$ an, so folgt die Abgeschlossenheit von $T^{n}(M)=T^{n+1}(X)$.

Satz 5. Ist $S \in L(X)$ ein Operator mit $T S=S T$ und $0 \in \varrho_{K}(T S)$, so folgt

$$
0 \in \varrho_{K}(T) \cap \varrho_{K}(S) .
$$

B e w e is. Es genügt, $0 \in \varrho_{K}(T)$ zu beweisen. Wegen $0 \in \varrho_{K}(T S)$ und $T S=S T$ erhalten wir zunächst

$$
N(T) \subseteq N(T S) \subseteq \bigcap_{n=1}^{\infty}\left(T^{n} S^{n}\right)(X) \subseteq \bigcap_{n=1}^{\infty} T^{n}(X)
$$

Wir zeigen nun die Abgeschlossenheit von $T(X)$. Dazu sei $\left(y_{n}\right)$ eine konvergente Folge in $T(X)$ und $y_{0}:=\lim _{n \rightarrow \infty} y_{n}$. In $X$ wählen wir eine Folge $\left(x_{n}\right)$ mit $T x_{n}=y_{n}$. Für die Folge $\left(S y_{n}\right)$ gilt dann

$$
S y_{n}=S T x_{n}=T S x_{n} \in(T S)(X) \text { und } \lim _{n \rightarrow \infty} S y_{n}=S y_{0} .
$$

Wegen der Abgeschlossenheit von $(T S)(X)$ erhalten wir $S y_{0} \in(T S)(X)=(S T)(X)$. Daher gibt es in $X$ ein $z_{0}$ mit $S y_{0}=S T z_{0}$. Der letzten Gleichung entnimmt man

$$
y_{0}-T z_{0} \in N(S) \subseteq N(T S) .
$$

Aus (1) ergibt sich dann $y_{0}-T z_{0} \in \bigcap_{n=1}^{\infty} T^{n}(X)$, insbesondere $y_{0}-T z_{0} \in T(X)$. Damit ist $y_{0} \in T(X)$ gezeigt. Der Bildraum $T(X)$ ist also abgeschlossen, woraus sich mit (1) schließlich $0 \in \varrho_{K}(T)$ ergibt.

\section{Der Spektralabbildungssatz. Er lautet so:}

Satz 6. Für jedes $f \in H(T)$ gilt $\sigma_{K}(f(T))=f\left(\sigma_{K}(T)\right)$.

B e w e is. Wir beweisen zuerst die Inklusion $f\left(\sigma_{K}(T)\right) \leqq \sigma_{K}(f(T))$. Dazu nehmen wir an, es sei $\lambda_{0} \notin \sigma_{k}(f(T))$ und zeigen, daß dann auch $\lambda_{0} \notin f\left(\sigma_{K}(T)\right)$ gilt. Angenommen, es sei $\lambda_{0} \in f\left(\sigma_{K}(T)\right)$. Aus dieser Annahme erhalten wir die Existenz eines $\mu_{0} \in \sigma_{K}(T)$ mit $f\left(\mu_{0}\right)=\lambda_{0}$. Die Funktion $g$ sei durch $g(\lambda):=\lambda_{0}-f(\lambda)$ erklärt. Dann gilt $g \in H(T)$ und $g\left(\mu_{0}\right)=0$, daher gibt es in $H(T)$ ein $h$ mit

$$
g(\lambda)=\left(\mu_{0}-\lambda\right) h(\hat{\lambda}) .
$$

Es folgt $g(T)=\left(\mu_{0} I-T\right) h(T)$. Aus $\lambda_{0} \in \varrho_{K}(f(T))$ ergibt sich

$$
0 \in \varrho_{K}(g(T))=\varrho_{K}\left(\left(\mu_{0} I-T\right) h(T)\right) .
$$


Mit Satz 5 erhalten wir daher

$$
0 \in \varrho_{K}\left(\mu_{0} I-T\right) \text {, also } \mu_{0} \in \varrho_{K}(T) .
$$

Dieser Widerspruch zu $\mu_{0} \in \sigma_{K}(T)$ zeigt uns $\lambda_{0} \notin f\left(\sigma_{K}(T)\right)$. Damit ist $f\left(\sigma_{K}(T)\right)$ $\subseteq \sigma_{K}(f(T))$ bewiesen.

Um die umgekehrte Inklusion $\sigma_{K}(f(T)) \subseteq f\left(\sigma_{K}(T)\right)$ zu zeigen, sei $\lambda_{0} \ddagger f\left(\sigma_{K}(T)\right)$. Wir setzen wieder $g(\lambda):=\lambda_{0}-f(\lambda)$. Dann ist $g(\lambda) \neq 0$ für jedes $\lambda \in \sigma_{K}(T)$. Besitzt $g$ in $\sigma(T)$ keine Nullstellen, so ist $g(T)=\lambda_{0} I-f(T)$ invertierbar, also $\lambda_{0} \in \varrho(f(T)) \subseteq \varrho_{K}(f(T)$, woraus wir $\lambda_{0} \notin \sigma_{K}(f(T))$ erhalten.

$\mathrm{Zu}$ betrachten ist also noch der Fall, daß $g$ in $\sigma(T)$ Nullstellen besitzt. Wegen Satz 3 hat $g$ in $\sigma(T)$ nur endlich viele Nullstellen $\lambda_{1}, \ldots, \lambda_{k}\left(\lambda_{i} \neq \lambda_{j}\right.$ für $\left.i \neq j\right)$ mit den Vielfachheiten $n_{1}, \ldots, n_{k}$. Wegen $g(\lambda) \neq 0$ für $\lambda \in \sigma_{K}(T)$ gilt

$$
\lambda_{i} \in \varrho_{K}(T) \text { für } i=1, \ldots, k .
$$

Mit einer geeigneten Funktion $h \in H(T)$, die auf $\sigma(T)$ nicht verschwindet, läßt sich $g$ in der Form

$$
g(\lambda)=\left(\prod_{i=1}^{k}\left(\lambda-\lambda_{i}\right)^{n_{i}}\right) h(\lambda)
$$

darstellen. Setzen wir abkürzend $p(\lambda):=\prod_{i=1}^{k}\left(\lambda-\lambda_{i}\right)^{n_{i}}$, so gilt

$$
g(T)=p(T) h(T)=h(T) p(T),
$$

wobei $h(T)$ in $L(X)$ invertierbar ist. Aus Hilfssatz 2 folgt daher

$$
N(g(T))=N(p(T))=N\left(\left(T-\lambda_{1} I\right)^{n_{1}}\right) \oplus \cdots \oplus N\left(\left(T-\lambda_{k} I\right)^{n_{k}}\right)
$$

und

$$
g(T)^{m}(X)=p(T)^{m}(X)=\bigcap_{i=1}^{k}\left(T-\lambda_{i} I\right)^{n_{i} m}(X)
$$

für jedes $m \in \mathbb{N}$. Mit der Abkürzung $D_{i}:=\bigcap_{m=1}^{\infty}\left(T-\lambda_{i} I\right)(X)$ erhalten wir dann

$$
\begin{aligned}
\bigcap_{m=1}^{\infty} g(T)^{m}(X) & =\bigcap_{m=1}^{\infty} \bigcap_{i=1}^{k}\left(T-\lambda_{i} I\right)^{n_{i} m}(X) \\
& =\bigcap_{i=1}^{k} \bigcap_{m=1}^{\infty}\left(T-\lambda_{i} I\right)^{n_{i} m}(X) \\
& =\bigcap_{i=1}^{k} \bigcap_{m=1}^{\infty}\left(T-\lambda_{i} I\right)^{m}(X)=\bigcap_{i=1}^{k} D_{i} .
\end{aligned}
$$

Wir zeigen nun

$$
N(g(T)) \subseteq \bigcap_{i=1}^{k} D_{i}
$$


Dazu sei $x \in N(g(T))$. Wegen (3) läßt sich $x$ in der Form $x=\sum_{i=1}^{k} x_{i}$ mit $x_{i} \in N\left(\left(T-\lambda_{i} I\right)^{n_{i}}\right)$ schreiben. Da jedes $\lambda_{i}$ zu $\varrho_{K}(T)$ gehört, folgt aus den Hilfssätzen 1 und 3 die Beziehung $x_{i} \in D_{j}$ für $i, j=1, \ldots, k$. Damit ist $x_{i} \in \bigcap_{j=1}^{k} D_{j}$ für jedes $i$, woraus $x \in \bigcap_{j=1}^{k} D_{j}$ folgt. Mit (5) erhalten wir dann

$$
N(g(T)) \leqq \bigcap_{m=1}^{\infty} g(T)^{m}(X)
$$

Mit Satz 4 und (4) ergibt sich schließlich noch die Abgeschlossenheit des Bildraumes $g(T)(X)$ und daraus mit (6)

$$
0 \in \varrho_{K}(g(T))=\varrho_{K}\left(\lambda_{0} I-f(T)\right),
$$

also $\lambda_{0} \notin \sigma_{K}(f(T))$. Somit haben wir auch $\sigma_{K}(f(T)) \subseteq f\left(\sigma_{K}(T)\right)$ gezeigt.

Zusatz bei der Korrektur.*) Nachdem die vorliegende Arbeit zum Druck angenommen wurde, ist eine Arbeit von M. Mbekhta (Résolvant généralisé et théorie spectrale, J. Operator Theory 21, 69-105 (1989)) erschienen, die einen Beweis des obigen Spektralabbildungssatzes enthält, allerdings nur für Operatoren auf Hilberträumen.

\section{Literaturverzeichnis}

[1] K. H. FöRSTER, Über die Invarianz einiger Räume, die zum Operator $T-\lambda A$ gehören. Arch. Math. 17, 56-64 (1966).

[2] H. Heuser, Funktionalanalysis. 2. Aufl., Stuttgart 1986.

[3] T. KaTo, Perturbation theory for nullity, deficiency and other quantities of linear operators. J. Analyse Math. 6, $261-322$ (1958).

[4] T. T. WeSt, A Riesz-Schauder theorem for Semi-Fredholm operators. Proc. Roy. Irish Acad. Sect. A 87, 137-146 (1987).

Anschrift des Autors:

Ch. Schmoeger

Universität Karlsruhe

Mathematisches Institut I

Englerstr. 2

D-7500 Karlsruhe 1

*) Eingegangen am 12.2. 1990. 